\title{
EFFECT OF SMOKING ON CARDIOVASCULAR SYSTEM
}

\author{
Biswajit Das ${ }^{1}$, Trinath Kumar Mishra ${ }^{2}$
}

${ }^{1}$ Associate Professor, Department of Cardiology, S. C. B. Medical College, Cuttack, Odisha.

2 Professor, Department of Cardiology, M.K.C.G. Medical College, Berhampur, Cuttack, Odisha.

\section{ABSTRACT}

\section{BACKGROUND}

Smoking is a major killer. It kills half of its users and lifetime smoker has $50 \%$ chance of smoking related death. Indians are heavy users of tobacco; people of lower socio-economic study commonly smoke bidis. Tobacco smoke contains 7000 compounds including phenols, carbohydrates and nitrosamines, many of which are carcinogenic. Nicotine, present in tobacco, is a vasoactive compound increasing vascular resistance. Acutely, smoking increases heart rate and BP within 10 minutes of exposure. There is a dose-response relationship between smoking and LV dysfunction. A Spanish study has shown smoking to be commonest risk factor for stroke. Cessation of smoking is the most cost effective strategy for CV disease prevention. Cessation of smoking reduces both MI and death irrespective of gender and duration of followup.

\section{KEYWORDS}

Smoking, Hypertension, Myocardial Infarction.

HOW TO CITE THIS ARTICLE: Das B, Mishra TK. Effect of smoking on cardiovascular system. J. Evolution Med. Dent. Sci. 2016;5(97): 7151-7154, DOI: $10.14260 /$ jemds/2016/1618

\section{BACKGROUND}

Smoking is the leading cause of preventable death and disease worldwide. Smoking kills about half of its users and a lifetime smoker has a $50 \%$ chance of dying due to smoking. Smokers on an average lose a full decade of their life as compared to $<3$ years with severe hypertension. ${ }^{1}$ While $1 / 3^{\text {rd }}$ of all tobacco related deaths are due to CVD (out of which CAD contributes $2 / 3^{\mathrm{rd}}$ ), $11 \%$ of all $\mathrm{CV}$ deaths and $30 \%$ of CAD related mortality is due to smoking. ${ }^{2}$ Smokers present with ACS 6 - 13 years earlier than non-smokers. Smoking is a strong independent risk factor of cardiovascular events and mortality even at older age, advancing cardiovascular mortality by more than five years, and smoking cessation in these age groups is still beneficial in reducing the excess risk.

More than 35\% of adults in India use tobacco, out of which the most common form is bidis (53\%) followed by cigarettes (19\%) and the rest in smokeless form. ${ }^{3}$ The interheart study demonstrated that all non-smoking forms of tobacco are equally potent aetiological factors in myocardial infarction. The prevalence of bidi smoking among Indian youth in the Global Youth Tobacco Survey (2000 - 2004) was 2.3\%, which increased to $3.5 \%$ in 2006.4 In a case control study conducted in Bangalore, it was found that the most important predictor of acute MI was current smoking of cigarettes or beedis. The odds of acute MI was 3.6 in current smokers overall and in individuals who currently smoked 10 or more cigarettes per day, it was 6.7 compared to never-smokers. Independent association was also found between beedi smoking and CHD risk with those consuming 25 beedis per day having a 10 -fold increased risk. A recent community-based survey in Punjab

Financial or Other, Competing Interest: None.

Submission 21-09-2016, Peer Review 23-11-2016,

Acceptance 29-11-2016, Published 05-12-2016.

Corresponding Author:

Dr. Trinath Kumar Mishra,

Professor, Department of Cardiology,

M.K.C.G. Medical College,

Bhabhanagar-2nd Line,

Berhampur-760004, Odisha.

E-mail: drtkmishra@yahoo.com

DOI: $10.14260 /$ jemds $/ 2016 / 1618$ found the prevalence of tobacco use to be $11.3 \%$ with a high incidence in adults aged 18 - 44 years and an average of 10 cigarettes per day for daily smokers. Tobacco smoking was more prevalent than smokeless tobacco consumption $(7.2 \%$ vs $5.2 \%) .5$ In a South Indian study of 820 patients of premature ACS ( $<40$ yrs.), tobacco consumption was the most important risk factor with a prevalence of $65 \%$ with hypertension and diabetes much less common $(<10 \%){ }^{6}$ The usage of smoked and non-smoked tobacco, while decreasing in more educated urban population continues to rise in rural and illiterate populations. ${ }^{7}$ A recent meta-analysis has provided evidence of geographical variation with significant positive association of smokeless tobacco with nonfatal IHD among users in Asia, but not in Europe. ${ }^{8}$

Tobacco smoke contains about 7000 compounds including phenols, phenols, carbonyls and nitrosamines that may be carcinogens; particulate matter such as tars; volatiles and gases such as carbon monoxide; and nicotine. ${ }^{9}$ Cigarette smoke or its individual components may cause insulin resistance and dyslipidaemia, promote vascular thrombosis and inflammation, induce aberrant vascular growth and angiogenesis and impair endothelial homeostatic and regenerative functions. Nicotine has 2 isomers-S (-) isomer is the most significant since it exerts a pleasant effect in the smokers, while Nicotine S (+) isomer, which has unpleasant but transient taste. Manufactured cigarette by Tobacco Industries has a reinforced power of the Nicotine S (-) isomer. Nicotine interacts with nicotinic receptors on vascular cells to promote angiogenesis. Nicotine is a vasoactive compound, which by increasing the activity of the sympathetic nervous system indirectly increases vascular resistance. Acutely, it increases release of catecholamines from adrenergic nerve terminals leading to an increase in heart rate and BP within 10 minutes of exposure. Oral intake of nicotine causes reduced heart rate variability representative of a shift of sympathovagal balance towards sympathetic activation. In the long term, tachyphylaxis to nicotine and its neural effects is quickly attained. "However, there is sympathetic hyperactivity in the long term as well in smokers. A number of putative mechanisms are implicated including activation of lung afferent $\mathrm{C}$ fibres, persistent attenuation of the inhibitory 
baroreflex and activation of peripheral arterial chemoreceptors. ${ }^{10}$

FMD represents a common parameter to measure endothelial dysfunction induced by smoking. Continuous smoking impairs FMD of the brachial artery in a dosedependent manner shown by the strong association between FMD and pack years smoked. Treatment of chronic smokers with tetrahydrobiopterin (Cofactor of NO synthase) improves FMD. ${ }^{11}$ Smoking increase total cholesterol, LDL, VLDL, triglycerides but reduces HDL and apoAl. Smoking cessation causes $4 \%$ increase in HDL without change in TG or LDL due to enhanced activity of LCAT and CETP. ${ }^{12}$ Smoking is proinflammatory as evidenced by an increase in WBC count, cytokines and CRP. Local pro-inflammatory effect is evidenced by increased expression of matrix metalloproteinases and inflammation. Smoking causes increased expression of adhesion molecules on endothelial cells, increased leucocyte number due to migration of leucocytes from bone marrow and increased incidence of infections (Chronic and acute exacerbations). A recent observational study showed that severity of smoking is associated with increased urinary F2isoprostane: creatinine ratio, MPO and WBC counts which improve after smoking cessation suggestion reduced inflammation. Smoking also promotes thrombosis by platelet activation, activation of coagulation system and inhibition of fibrinolysis. Smoking causes acute cardiovascular events by platelet activation and sympathetic hyperactivity leading to rise in heart rate, blood pressure and cardiac output. It also causes increased myocardial oxygen demand coupled with decrease in coronary artery diameter and increase in coronary resistance.

Smoking is a risk factor for development of type 2 DM. Smokers are hyperinsulinaemic and have greater accumulation of visceral fat. ${ }^{13}$ Diabetic patients who smoke have a higher incidence of microvascular and macrovascular complications, higher HbA1c and require more insulin. Genes influence smoking behaviour, metabolism of chemicals in smoke and the atherogenic potential of these compounds. Studies have shown glutathione S-transferase theta genotype GSTT1-1 in heavy smokers predisposes to pre-clinical atherosclerosis in the form of increased intima-media thickness. Also, CYBA gene A640G polymorphism modifies severity of CAD through interactions with smoking and hypercholesterolaemia. ${ }^{14}$ Smokers have a greater incidence of SCD than non-smoker.

The relative risk of SCD in smokers is much higher than for MI and the incidence of smoking is higher in the SCD population than the non-SCD population. Moreover, this risk decreases rapidly after cessation indicating that acute effects of tobacco exposure are probably causative. Smoking is the third most important risk factor for heart failure (After DM and CHD, both of which are caused by smoking) increasing the risk by $60 \%$. Echocardiographic studies show that there is a dose response relationship between smoking and impairment of LV structure and function. Longer duration of smoking was associated with increased LV mass, worse LV systolic (Ejection Fraction) and diastolic function (E/e'). Smoking is also causative in extra cardiac vascular disease including stroke (ischaemic stroke and subarachnoid haemorrhage) and aortic aneurysm. The incidence rate (per 100,000) of stroke among a cohort of 118,538 women was $49.8 \%$ for smokers as compared to $17.7 \%$ for non-smokers. A retrospective Spanish study of young stroke patients (Both ischaemic and haemorrhagic) found smoking to be the most common risk factor (55\%). In a stroke registry study in Hyderabad, $28 \%$ of patients with ischaemic stroke had a history of smoking. ${ }^{15}$ Smoking is a much stronger risk factor for abdominal aortic aneurysm both in causation and in progression and risk of rupture of established AAA. Smoking may activate tissue plasminogen activator, which is a potent activator of elastolytic matrix metalloproteinase. ${ }^{16}$ This could link smoking to aortic elastolysis, which is the central pathologic component of aortic aneurysm. Smoking has a strong causative role in peripheral artery disease, both atherosclerotic and non-atherosclerotic (Thromboangiitis obliterans), responsible for $90 \%$ of cases in the non-diabetic population. In a study conducted in Bangladesh, those smoking 11 - 20 beedis per day were 7 times more likely and those smoking $>20$ beedis per day were 34 times more likely to have TAO compared to those smoking $<10$ cigarettes per day. It also causes Raynaud's phenomenon. Ischaemic ulcers are more resistant to treatment in smokers.

\section{HTN and Smoking}

Smoking has been shown to cause sympathetic nervous system stimulation, activation of RAS and reduced NO synthesis, all of which contribute to hypertension. Activation of RAS leads to release of angiotensin II contributing to both hypertension and endothelial dysfunction. Smokers and former smokers have increased vascular stiffness as assessed by pulse wave velocity and wave reflection, which are reversed after a decade of cessation. Smoking is detrimental to hypertension, especially in those who smoke $>15$ cigarettes per day and in those with history of CAD or heart failure. However, clinical studies have not shown a consistent relationship between hypertension and smoking with strong correlation only in certain subgroups such as elderly smokers. A recent hospital-based observational study in Kerala displayed a strong association between hypertension and smoking with the incidence of current smokers among smokers being more than twice that in non-smokers (16.9 vs $7.5 \%) .17$

\section{Effect of Smoking on Therapy}

In a seemingly contradictory observation, it was seen that there was a reduced mortality for smokers with STEMI. Thrombolysis was more effective as evidenced by greater incidence of TIMI 3 flow. Based on data from randomised studies and a large meta-analysis, there is a substantial cardiovascular event reduction with clopidogrel therapy in smokers, but not in non-smokers due to cytochrome P450 (CYP) 1A2 and CYP2B6 induction by cigarette smoking resulting in greater clopidogrel Active Metabolite (AM) generation. Post hoc analysis of CAPRIE trial showed that clopidogrel is more efficacious than aspirin when used for secondary prevention including reduction in ML and death. However, the more potent ticagrelor and prasugrel have the same efficacy in all patients irrespective of smoking status. In patients with reperfused STEMI, CMR showed a strong direct relationship between intramyocardial haemorrhage (Indicative of reperfusion injury) and smoking, but it also revealed an inverse relationship of smoking with adverse LV remodelling which was nullified in patients with IMH underlining the importance of mitigation of reperfusion injury 
in smokers. Analysis of smoker subgroup of PLATO showed that after correction for baseline characteristics, the risk of cardiovascular events did not differ between smokers and ex/non-smokers except for stent thrombosis, which was higher in smokers. A 5-year follow-up of SYNTAX showed that smoking is associated with worse outcomes after revascularisation (Both $\mathrm{CABG}$ and $\mathrm{PCI}$ ) in patients of complex CAD due to greater incidence of recurrent MI. Other studies have shown that smoking status before Coronary Artery Bypass Graft (CABG) surgery does not affect survival after surgery, but persistent smokers after CABG have a greater relative risk of all-cause mortality, cardiac death and need for repeat revascularisation compared to those who stopped smoking for at least one year. Smoking is associated with lower 30 day mortality, but higher long-term mortality after MI. Current smoking is associated with large number of life years lost after MI. Hence, there are contradictory data and it appears that the smokers have a lower mortality after MI due to their younger age and less incidence of comorbidities. ${ }^{18}$ This has again been demonstrated in a sub-study of IABP-SHOCKII trial where smokers had lower mortality after univariable analysis, but not after adjustment of important confounders like diabetes and hypertension. Smoking has been shown to have a strong correlation with stent fracture in self-expanding nitinol stents used for femoro-popliteal obstructive disease possibly due to vessel calcification and inelasticity.

Worldwide, $40 \%$ of children, $33 \%$ of non-smoking men and $35 \%$ of non-smoking women are victims of passive smoking. Second hand smoke is responsible for $6,00,000$ deaths per year (Including 3,90,000 due to ischaemic heart disease) accounting for $1 \%$ of the global mortality. ${ }^{19}$ Regular exposure to second hand smoke confers a $25 \%$ increased risk of a cardiac event as compared to $78 \%$ in a heavy smoker $(20$ cigarettes/day), although the proportional tobacco exposure is only $1 \%$. This disproportionately high risk is explained by the highly nonlinear dose effect relationship of tobacco smoke - highly incremental effects at low doses, which saturate at higher doses. Moreover, second hand smoke may be more toxic per gram of total particulate matter than the "mainstream" cigarette smoke that a smoker inhales. SHS has also consistently been shown to be associated with an increased incidence of stroke (RR of 1.35). Strict implementation of smoke free laws has resulted in $14-17 \%$ reduction in MI after 1 year in multiple studies. ${ }^{20}$ Third hand smoke refers to the contamination of surfaces such as carpets and curtains with components of tobacco smoke after the cigarette is extinguished and its contents have been cleared from the air. The fabrics may catalyse further oxidation of smoke components. Preliminary studies suggest that these components may have adverse health effects including cardiovascular effects. ${ }^{21}$

Stopping smoking is the most cost-effective strategy for CVD prevention. For secondary prevention, cessation of smoking has been demonstrated to significantly reduce both death and MI irrespective of gender, duration of follow-up or study site. Benefits of quitting appear within days of abstinence. Within 2 months, BP and heart rate normalise and by 6 months arterial stiffness and endothelial function improve. While significant morbidity benefits accrue over 6 months the risk of death, MI and stroke approach that of nonsmoker by 5 years and by 20 years all haemostatic and inflammatory markers reach the baseline along with regression of atherosclerosis in peripheral arteries. However, in the Euroaspire IV survey among CAD patients, $16 \%$ smoked after a mean follow-up time of 16 months and nearly half of the participants who smoked at the time of their coronary event were persistent smokers. A recent Danish registry study of young CAD patients found smoking to be the most common risk factor at disease onset (72\%), but more importantly at a median of 5.6 years followup, $37 \%$ of patients continued to smoke underlining the insufficient secondary prevention measures. Both high-sensitivity C-reactive protein $>3 \mathrm{mg} / \mathrm{L}$ and particularly CAC $>100$ identified high-risk smokers who may benefit from more intensive smoking-cessation efforts. A 4-step non-invasive ultrasound assessment protocol, ABCD may be useful for detection and monitoring of early arterial damage in smokers (A, the Ankle-Brachial Index (ABI); B, the Breath Holding Index (BHI); $\mathrm{C}$, the Carotid Intima Media Thickness (CIMT); and D, the Diameter of the abdominal aorta). ${ }^{22}$ Following the failure of advice, encouragement and motivational interventions or in addition to them NRT varenicline or bupropion should be offered to assist cessation. Electronic cigarettes deliver the addictive nicotine without the vast majority of tobacco chemicals and are probably less harmful than tobacco. Studies show that e-cigarettes are moderately effective as smoking cessation and harm reduction aids, but that a significant component of that effect is due to changes in behaviour rather than in nicotine delivery. An average weight gain of $5 \mathrm{~kg}$ on cessation of smoking is due to increased caloric intake, reduced heart rate and improved lipoprotein lipase activity and is not associated with increased coronary events.

It is estimated that at the current rate, 1 billion deaths would occur in the $21^{\text {st }}$ Century due to tobacco use and by the year 2030 one in every six individuals will die because of the ill effects of tobacco. ${ }^{23}$ Faced with such grim statistics, it is imperative to redouble our efforts for smoking cessation for both primary and secondary prevention including patient counselling and pharmacological measures where required.

\section{REFERENCES}

1. Doll R, Peto R, Boreham J, et al. Mortality in relation to smoking: 50 years observations on male British doctors. BMJ 2004;328:1519.

2. Prescott E, Hippe M, Schnohr P, et al. Smoking and risk of myocardial infarction in women and men: longitudinal population study. BMJ 1998;316(7137):1043-7.

3. Gupta R, Gupta N, Khedar RS. Smokeless tobacco and cardiovascular disease in low and middle income countries. Indian Heart J 2013;65(4):369-77.

4. Gupta R, Mohan I, Narula J. Trends in coronary heart disease epidemiology in India. Annals of Global Health 2016;82(2):307-15.

5. Thakur JS, Jeet G, Pal A, et al. Profile of risk factors for noncommunicable diseases in Punjab, northern India: results of a state-wide STEPS survey. PLoS ONE 2016;11(7): e0157705.

6. Zarin P, Shaffi M. Modifiable risk factors of hypertension: a hospital-based case-control study from Kerala, India. Journal of Family Medicine and Primary Care 2016;5(1):114-9. 
7. Oommen AM, Abraham VJ, George K, et al. Prevalence of coronary heart disease in rural and urban vellore: a repeat cross-sectional survey. Indian Heart J 2016;68(4):473-9.

8. Vidyasagaran AL, Siddiqi K, Kanaan M. Use of smokeless tobacco and risk of cardiovascular disease: a systematic review and meta-analysis. Eur J Prev Cardiol 2016;pii:2047487316654026.

9. Leone A. Toxics of tobacco smoke and cardiovascular system: from functional to cellular damage. Current Pharmaceutical Design 2015;21(30):4370-9.

10. Cooke JP. New insights into tobacco-induced vascular disease: clinical ramifications. Methodist Debakey Cardiovasc J 2015;11(3):156-9.

11. Messner B, Bernhard D. Smoking and cardiovascular disease: mechanisms of endothelial dysfunction and early atherogenesis. Arterioscler Thromb Vasc Biol 2014;34(3):509-15.

12. Jaana K, Ventola H, Jokelainen J, et at. Cardiovascular disease risk factors in relation to smoking behaviour and history: a population-based cohort study. Open Heart 2016;3(2):e000358.

13. Keith RJ, Al Rifai M, Carruba C, et al. Tobacco use, insulin resistance, and risk of type 2 diabetes: results from the multi-ethnic study of atherosclerosis. PLoS ONE 2016;11(6):e0157592.

14. Brieitling LP. Current genetics and epigenetics of smoking/tobacco-related cardiovascular disease. Arterioscler Thromb Vasc Biol 2013;33(7):1468-72.
15. Amalakanti S, Nagariunakonda S, Uppala V, et al. Stroke: changing risk factor profile and unaltered mean age in a developing country. a retrospective study and literature review. Eur Neurol 2016;75(5-6):229-35.

16. Norman PE, Curd JA. Understanding the effects of tobacco smoke on the pathogenesis of aortic aneurysm. Arterioscler Thromb Vasc Biol 2013;33(7):1473-7.

17. Prabhakaran D, Jeemon $P$, Roy A. Cardiovascular diseases in india: current epidemiology and future directions. Circulation 2016;133(16):1605-20.

18. Kirtane AJ, Kelly CR. Clearing the air on the smokers paradox. J Am Coll Cardiol 2015;65(11):1116-8.

19. Fischer F, Kraemer A. Meta-analysis of the association between second-hand smoke exposure and ischaemic heart diseases, COPD and stroke. BMC Public Health 2015;15:1202.

20. Tan CE, Glantz SA. Smoke-Free air: an important strategy to reduce heart attacks. Global Heart 2012;7(2):189-91.

21. Northrup TF, Jacob P, Benowitz NL, et al. Thirdhand smoke: state of the science and a call for policy expansion. Public Health Rep 2016;131(2):233-8.

22. Mozzini C, Casadei A, Roscia G, et al. Young smoker ABCD vascular assessment: a four-step ultrasound examination for detecting peripheral, extra and intra-cranial early arterial damage. BMC Cardiovascular Disord 2016;16:147.

23. Seligman B, Vedanthan R, Fuster V. Acute coronary syndromes in low- and middies-income countries: moving forward. Int J Cardiol 2016;217 Suppl:S10-2. 